\title{
Comportamiento del ReCAudo \\ DEL IMPUESTO DEPARTAMENTAL AL \\ DEGÜELLO DE GANADO MAYOR EN \\ el Municipio de Montería \\ EN LOS AÑOS 2OI 3-20I $8^{1}$
}

\section{DEPARTMENTAL TAX ON THE}

SLAUGHTER OF CATTLE IN

Montería Municipality,

2OI 3-20 I 8

\author{
Laura Vanessa Castrillón Galeano ${ }^{2}$ \\ Mariangela Ramos de la Ossa ${ }^{3}$
}

1 Este artículo es resultado del trabajo de grado que las autoras presentaron en la Maestría en Tributación y Política Fiscal, ofrecido por la Universidad de Medellín en la ciudad de Sincelejo. El programa se ofrece allí en convenio con la Universidad de Sucre. Fue orientado por los profesores de la Universidad de Medellín Hugo A. Macías y Robinson Rivera, desde el punto de vista metodológico y temático, respectivamente.

2 Abogada, Universidad Pontificia Bolivariana Montería. Magíster en Tributación y Política Fiscal. Medellín, Colombia. Correo electrónico: laura-vane3215@hotmail.com Orcid: https://orcid.org/0000-0002-4636-1544

3 Contadora pública, Corporación Universitaria del Caribe (CECAR), Sincelejo. Magíster en Tributación y Política Fiscal, Universidad de Medellín. Medellín, Colombia. Correo electrónico: madeoss-94@hotmail.com

Orcid: https://orcid.org/0000-0003-2840-6654

Código JeL: H26, H71

Fecha de recepción: 17/03/2020

Fecha de aceptación: 29/04/2020

DOI: https://doi.org/10.18601/16577175.n27.04 


\section{Resumen}

En la comunidad cordobesa no existe desconocimiento de que en el territorio concurre mucha informalidad en el ejercicio del degüello de ganado, dado que no todos los ganaderos sacrifican el ganado en plantas, mataderos o frigoríficos legalmente constituidos, pues realizan estas labores en las fincas donde crían el animal, filtrando y evadiendo este impuesto, generando un recaudo bajo y poco significativo para el Departamento. En este artículo se propone evaluar la eficiencia de la gestión del recaudo que adelanta el Municipio de Montería en los ańos 2013-2018 sobre el impuesto al degüello de ganado mayor; como estrategia metodológica se implementaron muestras cuantitativas, se solicitó información a la Secretaría de Hacienda Departamental, sobre las finanzas recaudadas año por año de 2013 a 2018 en concepto del impuesto en mención y muestra cualitativa, se realizaron entrevistas a diferentes sujetos que intervienen en el tema abordado. Como resultado, se identificaron las desventajas en la fiscalización del impuesto al degüello de ganado mayor y en las conclusiones se propone la estrategia que han implementado muchos municipios en Colombia para mejorar los procesos de recaudo y mitigar la evasión de los tributos.

Palabras clave: ganado mayor; impuesto al degüello; impuestos municipales; recaudo; rentas departamentales.

\section{Abstract}

In the Cordovan community there is no ignorance that in the territory there is a lot of informality in the exercise of the slaughter of cattle, since not all the cattlemen slaughter the cattle in legally constituted plants, slaughterhouses or cold stores, as they carry out these tasks on the farms where they breed the animal, filtering and evading this tax, generating a low and insignificant collection for the Department. This article proposes to evaluate the efficiency of the collection management carried out by the Municipality of Montería in the years 2013-2018 on the slaughter cattle tax. As a methodological strategy, quantitative samples were implemented, information was requested from the Department of Departmental Finance, on the finances collected year by year from 2013 to 2018 for the mentioned tax and qualitative sample, interviews were conducted with different subjects involved in the subject boarded. As a result, the main strategies to improve the processes of inspection and implementation of the collection of all the taxes established in the Departmental.

Key words: departmental income; municipal taxes; collection; slaughter tax; major cattle. 


\section{INTRODUCCIÓN}

En virtud de la Ley 8 de 1909 y el Decreto 1222 de 1986, código de renta departamental, se crea el impuesto al degüello, el Departamento de Córdoba adoptó la normatividad en cuanto a los impuestos como determinación, liquidación, cobro y el procedimiento. La Ordenanza 07 de 2012, en su artículo cuarto capítulo I título I, faculta a la Secretaría de Hacienda Departamental, la gestión, el recaudo, fiscalización, determinación, discusión, devolución y cobros de los tributos departamentales. El capítulo XIV está referido al impuesto al degüello de ganado mayor y dispone de 17 capítulos donde determina los elementos del tributo, sujeto activo, sujeto pasivo, hecho generador, base gravable y tarifa.

Uno de los aspectos importantes del impuesto al degüello de ganado mayor lo establecen la Ordenanza 07 de 2012 (art. 153) y la Ordenanza 07 de 2012 (art. 157) del Estatuto de Rentas departamentales de Córdoba que fija el hecho generador y base gravable del impuesto: "[...] está constituido por el sacrificio de cada unidad de ganado mayor, bovino, bufalino y equino, realizados por plantas de beneficios, mataderos públicos, cooperativas y frigoríficos legalmente constituidos; y su base gravable lo constituye cada unidad de ganado mayor que se sacrifique". En Colombia se encuentran varios textos que desarrollan el tema. En el caso del Municipio de Florencia, los autores evalúan la eficiencia en el recaudo del impuesto al degüello de ganado mayor en los periodos fiscales 2009-2010; en el caso del Municipio de Quibdó, hacen una propuesta metodológica para aumentar el recaudo tributario municipal; y en el caso del Departamento de Chocó, describen una estrategia para el mejoramiento del recaudo del impuesto al degüello de ganado mayor en el Departamento. Estas investigaciones permiten ver cómo se ha desarrollado el contexto de descentralización territorial en las administraciones locales en diferentes partes del país. Una de las propuestas metodológicas para aumentar el recaudo tributario en el ámbito local es la implementación de metodologías de tecnologías de información y técnicas de gestión, para acercarse al contribuyente (Farfán \& Mosquera, 2015); sin embargo, las administraciones locales han presentado fuertes inconvenientes con la eficiencia en el aumento del recaudo del impuesto al degüello de ganado mayor, por falta de gestión y capacitación de los contribuyentes, presentándose en el Municipio de Florencia y muchos municipios del país que se dedican a la explotación ganadera (Restrepo, Hernández \& Moreno, 2013).

Como profesionales con maestría en el área tributaria, en la investigación realizada para escribir este artículo se adquirieron y afianzaron conocimientos respecto a la administración local, permitiendo proponer alternativas de solución a problemas específicos en el manejo de las rentas territoriales del Municipio de Montería por concepto de degüello de ganado mayor; según nuestra investigación en textos académicos de varios lugares del país y la práctica de campo realizada en Montería a diferentes intervinientes de la actividad ganadera, se puede decir que es una problemática general del territorio colombiano, pues cada secretaría de Hacienda del país debe trabajar en mejorar su gestión y control del recaudo de las finanzas públicas. 
Con el fin de mitigar el impacto de la evasión de impuestos en el país, en algunos departamentos, como Antioquia, Cundinamarca y Valle del Cauca, se ha añadido en sus ordenanzas departamentales la creación del grupo operativo anticontrabando de rentas. El aporte que hacemos a la comunidad cordobesa es proponer a la Secretaría de Hacienda la creación del grupo anticontrabando o anticorrupción para que las rentas del Departamento se fortalezcan debido a que son muy afectadas por la evasión y se dejan de percibir ingresos importantes, que podrían destinarse en áreas como educación y bienestar social. De esta manera invitar a todas las alcaldías y gobernaciones acogerse a la creación de este grupo en su ordenanza para empezar a reducir los efectos, e implementen y preparen en su plan de desarrollo políticas públicas para luchar en contra de este flagelo.

El objetivo general del artículo es evaluar la eficiencia del recaudo del impuesto al degüello de ganado mayor en el Municipio de Montería en los años 2013-2018, es decir, con base en los resultados generados se espera determinar si la gestión que adelanta la Secretaría de Hacienda Departamental de Córdoba es eficiente en el recaudo por concepto del impuesto al degüello de ganado mayor en el Municipio de Montería, y así analizar si ha aumentado progresivamente los últimos cinco años o cuál ha sido el comportamiento de ese recaudo. Para lograrlo se analizó el impuesto aplicado y su potencial de recaudo, se determinó cuáles son los sujetos pasivos del impuesto, se requirió a la Secretaría de Hacienda del Departamento de objeto de estudio información sobre los recaudos y se realizó entrevistas a varios participantes del impuesto en mención. Además de esta introducción, en el segundo acápite presentamos nuestro marco de referencia (revisión de literatura y bases conceptuales del impuesto territorial de estudio), luego describimos la metodología utilizada, exponemos los resultados y finalmente presentamos las conclusiones y proponemos una serie de estrategias a implementar en el Municipio, para fortalecer el recaudo de sus ingresos tributarios.

\section{REVISIÓN DE LITERATURA}

En este artículo se realiza una exploración de investigaciones académicas referentes al tema de investigación del trabajo, el cual es el impuesto al degüello de ganado mayor; para la elección de los artículos académicos se realizó una búsqueda en las diferentes revistas académicas, tomadas como referencias.

El degüello de ganado es una actividad realizada con fines de abastecimiento de carnicerías destinada al consumo humano que legalmente debe realizarse en frigoríficos o mataderos que cuenten con todas las condiciones de sanidad, salubridad y permisos exigidos para su funcionamiento. Este es una actividad que está grabada por el Departamento cuya causación se da al momento de sacrificar el ejemplar (Hoyos, 2016).

Frigosinú es el principal sujeto pasivo del impuesto al degüello de ganado mayor en el Municipio de Montería, es una empresa legalmente constituida y registrada en Cámara de Comercio con el NIT 800052046-3, cuenta con permisos y registros 
sanitarios para sacrificar ganado a otras empresas; también procesa alimentos, subproductos del desposte de ganado bovino y bufalino tales como: embutidos, vísceras, cebos, harinas de sangre, harinas de carne, entre otros, cuyos productos son expendidos al mercado nacional e internacional (Pérez, 2016).

En el año 1993 la planta Frigosinú tuvo crisis y estuvo al borde de cerrar; el gerente de la empresa en ese entonces, Gustavo Mejía, anunció que el matadero seguiría trabajando a media máquina, realizando sacrificio de sesenta reses diarias que se consumen en Montería, donde sacrificaban mensualmente 2500 reses de propiedad privada y 1600 para el consumo del municipio costeño. Mejía señaló que la principal causa de esta crisis se origina en que las exportaciones de carne para los mercados de Venezuela y Perú fueron suspendidas desde agosto de 1991, por problemas de cambio de moneda. El número de reses sacrificadas no respondía a los costos financieros y operativos de la empresa, situación que se agravó por la competencia desleal que generan los mataderos clandestinos (El Tiempo, 1993); crisis que con el pasar de los años se superó y es una empresa que actualmente opera con normalidad y en la ciudad de montería.

Con el proceso de las reformas constitucionales de los años ochenta y noventa se producen cambios en pro de modernizar el Estado; con la reforma constitucional del 91 se reforzó la descentralización territorial en nuestro país con el propósito que las entidades territoriales manejaran sus propias normas fiscales (Betancur, 2007).

Con las ordenanzas departamentales se regulan la aplicación, el control y las sanciones de los impuestos locales y es manejado por los departamentos gracias a la autonomía que le otorga la legislación colombiana, para que las administraciones departamentales implementen estrategias dirigidas a aumentar sus ingresos para reinvertirlos (Núñez, 2005; Talero \& Quintero, 2011; Lindo, 2016).

Los impuestos departamentales son una de las fuentes de financiación más importantes de estas entidades territoriales, el estudio de estos tributos es limitado, pero en varias investigaciones académicas se aborda el comportamiento de los impuestos locales. En este texto se revisa la estructura y el efecto de las rentas del Departamento de Antioquia, evaluando e implementando diferentes políticas, con el propósito de anticipar los posibles resultados y de este modo proponer acciones concretas para el mejoramiento del recaudo (López, 2014).

En esta investigación se analizaron las características del comportamiento tributario de los ingresos propios municipales de Ocańa, en el Estatuto Municipal estableció todas las rentas e impuestos del Municipio, así como el proceso de recaudo del impuesto predial y los demás impuestos municipales que se perciben; donde el propósito central es describir las razones sobre el no pago y evasión de los impuestos generados por la inestabilidad tributaria a través de los años (Vásquez \& Jiménez, 2015; Coronel \& Pérez, 2015; Carrascal, 2015). En Antioquia además de la ganadería se realiza exportación bananera, se resalta la actividad de una finca bananera ubicada en la zona de Urabá bajo la metodología flujo de caja libre, la cual se encontró que la finca está valorada en promedio \$13.382.277.080,70 y máxima de \$14.051.390.934,74 (Henao \& Caro, 2019). 
Además de la evasión de impuestos y el contrabando, existe otro flagelo que afecta instituciones públicas y privadas en el desarrollo social y económico, el cual es la corrupción, que influye en el comportamiento de los indicadores económicos, PIB, desempleo y competitividad en sus niveles (alto, medio y bajo) (Aldana, Alonso \& Albarracín, 2019).

En el Municipio de Quibdó unas funcionarias realizaron un proyecto en el cual proponen diferentes medidas y estrategias para fortalecer el sistema tributario, que permitan lograr un aumento de ingresos propios, debido a que los ingresos recaudados son bajos e insuficientes para el financiamiento de los gastos de funcionamiento del Municipio (Farfán \& Mosquera, 2015).

En el Departamento de Chocó se evidenció el panorama sobre el comportamiento de una de sus rentas, el de degüello de ganado mayor, este es representativo dentro del presupuesto de ingresos que ha venido presentando disminución considerable frente a las otras rentas, con la que se propone tomar las medidas necesarias para mejorar el crecimiento de este impuesto (Perea, Palacios, \& Rentería, 2018).

En esta investigación se hace una descripción general de la Gobernación del Tolima los municipios que están declarando el sacrificio de cabeza de res, la Secretaría de Hacienda con el fin de dinamizar la cartera del Departamento recoge para las arcas impuestos de varias índoles: consumo cigarrillo, consumo de alcohol, vehículo y degüello de ganado; este último impuesto en el Departamento de Tolima obtuvo un recaudo de \$4.375.158.399 en los últimos cinco años (Ardila, 2019).

En el Municipio de Río Sucio (Cesar) el impuesto de degüello de ganado mayor hace parte de los impuestos de mediano y menor recaudo, otros recaudos de esta administración municipal relacionados con la ganadería son: coso municipal (es el valor que se cobra por la tenencia o albergue transitorio de animales que se encuentren en vía pública o predios ajenos al ser reclamados por su propietario pasados 30 días), registro y custodia de patentes, marcas y herretes (hierro con el que se marca el ganado para identificarlo) (Mosquera, 2014).

En esta investigación realizaron análisis de los tributos del orden Departamental del Triángulo del Café, donde se puedo evidenciar que el Departamento del Quindío es el más atractivo para la inversión de las empresas, tarifas bajas en la mayoría de sus impuestos el primer lugar es el impuesto de registro con una tarifa del 0,3\% sobre registro de los actos o contratos con cuantía en cámaras de Comercio (López \& Martínez, 2011).

El desconocimiento de las diferencias del código de rentas de los municipios de Pereira y Dosquebradas se genera por la falta de actualización de los contribuyentes y la poca formación y capacitación que reciben los funcionarios competentes; Pereira tiene más impuestos que el Municipio de Dosquebradas pero en Dosquebradas se manejan tarifas más bajas para el cálculo de los impuestos, en estas entidades deben mantener un Código de Rentas actualizado (Loaiza, Cardona \& Quintero, 2012).

Esta investigación en materia de recaudo de impuesto de degüello en el Municipio de Florencia permite conocer su comportamiento y tener referencia para que las entidades competentes tomen medidas de acción para mejorar la operatividad 
de este impuesto en el Departamento, ya que predomina la actividad ganadera (Restrepo, Hernández \& Moreno, 2013).

Este trabajo se basa en la elaboración de prácticas y estrategias para el mejoramiento del recaudo de impuestos del Municipio de Arauca, donde llegaron a la conclusión de que deben elaborar ordenanzas tributarias de manera clara y precisa que establezcan los elementos constitutivos del tributo, así como los plazos y formas de declaración del impuesto (Valencia, 2017).

Analizando otros aspectos de la ganadería, el aprovechamiento de materias primas naturales que se extraen del ganado bovino como son los cuernos de este animal, en el Municipio de Tierra Alta (Córdoba) además de realizar sacrificio de ganado se utiliza el cuerno para confeccionar artesanías especialmente de bisutería (González \& Salazar, 2008).

Desde hace varios años el sector ganadero comenzó a tener crisis de rentabilidad, debido a efectos de los tratados de libre comercio, cambios climáticos, el alza de los insumos, la disminuida capacidad industrial y ausencia de inversión pública; por lo anterior, el Gobierno Nacional comenzó a implementar "Pacto Nacional por el Agro y el Desarrollo Rural" para mejorar la labor de los 500 mil ganaderos del país (Fedegan, 2019).

En los trabajos a los que hemos hecho referencia es evidente la necesidad de que en los departamentos y municipios del país la administración local implemente estrategias orientadas a capacitar y controlar las actividades ganaderas de los posibles contribuyentes, pues en algunos casos se evade el impuesto por desconocerlo y en otros por falta de sanción a quienes realizan prácticas clandestinas e ilegales sin cumplir los mínimos de salubridad y sanidad.

A pesar de los operativos que realizan las autoridades sigue sin freno el sacrificio ilegal en muchas regiones del país, los productores se quejan por el aumento del sacrificio ilegal de ganado, situación que no solo afecta a la salud pública sino que perjudica los intereses económicos de cientos de productores que realizan el faenado en lugares autorizados; estos pagan el impuesto y deben incurrir en más gastos (Contexto Ganadero, 2013).

\section{Metodología}

La presente investigación es de carácter descriptivo porque con la metodología empleada se investigó y se recolectaron datos reales y precisos del impuesto departamental de degüello ganado mayor en el Municipio de Montería para el periodo 2013-2018; y es exploratoria porque es un tema con delimitado conocimiento. Se pretendió determinar cuáles son los establecimientos, plantas de beneficios, mataderos y frigoríficos constituidos en la ciudad de Montería, es decir, los sujetos pasivos del impuesto. El impuesto al degüello de ganado mayor es de orden departamental, por ende el Departamento puede fijar libremente la tarifa del impuesto, y es el sujeto activo encargado de administrar, recaudar y cobrar; el propósito de la investigación es evaluar la eficiencia del recaudo de esta renta. 
Los procedimientos de recolección de datos para esta investigación se basaron en solicitar a la Secretaría de Hacienda Departamental de Córdoba, en el área de fiscalización, las estadísticas de los recaudos del impuesto al degüello de ganado mayor en el Municipio de Montería de los últimos cinco años; el otro procedimiento que se utilizó fue la aplicación de una entrevista dirigida a diferentes intervinientes del tema en cuestión, a Frigosinú como sujeto pasivo de este impuesto, la Corporación Autónoma Regional del Valle del Sinú y el Río San Jorge (cvs), la Policía Ambiental como autoridades que controlan el ejercicio de degüello, el Instituto Colombiano Agropecuario (ICA), el Instituto Nacional de Vigilancia de Medicamentos y Alimentos (Invima) y la Secretaría de Salud.

Con los instrumentos mencionados anteriormente, se analizó la información suministrada por la Secretaría de Hacienda Departamental, evaluando la eficiencia de las políticas empleadas para la fiscalización, el control y la forma en que se recauda el impuesto, el cumplimiento de las normas tributarias por parte de los contribuyentes de este impuesto (Frigosinú) y así mismo resaltar que existen sitios que sacrifican ganado de forma ilegal y sin las condiciones mínimas de salubridad, haciendo comparación y observaciones sobre los resultados obtenidos de las entrevistas realizadas a los funcionarios de la Oficina de Rentas Departamentales, cvs, ICA, Invima, Frigosinú, Secretaría de Salud y la Policía Ambiental de Montería.

La población de esta investigación se encuentra delimitada en los mataderos, plantas de beneficio, frigoríficos públicos o privados legalmente constituidos, la Secretaría de Hacienda Departamental y las entidades ambientales y sanitarias (Policía Ambiental y cvs).

En la metodología se tenía planeado entrevistar a la Corporación Autónoma Regional del Valle del Sinú y Río San Jorge (cvs), Instituto Colombiano Agropecuario (ICA), Instituto Nacional de Vigilancia de Medicamentos y Alimentos (Invima), Frigosinú, Policía Ambiental, Secretaría de Salud; con fines de resolver los objetivos de este artículo. La información obtenida en la Secretaría de Hacienda Departamental sobre el recaudo del impuesto al degüello de ganado mayor en el Municipio de Montería se analizará para estimar el progreso de dichos ingresos al Departamento. En esta investigación también fue necesario utilizar fuentes de datos secundarias como investigaciones y artículos en los que se analizó el impuesto al degüello de ganado mayor en otros municipios y departamentos de Colombia, referencias e información de internet, sitios web (Ordenanza 07, 2012), ordenanzas de rentas departamentales de Córdoba, Constitución Política de Colombia y Ley 8 de 1909, la cual estableció los primeros preceptos legales en materia de rentas departamentales.

\section{Resultados y Discusión}

Se puede observar en el siguiente gráfico que desde el año 2014 al 2019 el recaudo del impuesto fue aumentando significativamente, reflejando una variación positiva del 10,13\%. Según la información suministrada por la Secretaría de Hacienda de Córdoba, el incremento respondió a la actividad económica de los frigoríficos de 
Ciénaga de Oro y Montería, así mismo aumentaron las exportaciones del producto cárnico; del periodo del 2019 se puede inferir que el recaudo del impuesto es menor con respecto al del 2018 debido que se analizó la cifra recaudada hasta el mes de septiembre del 2019, que era el cursado cuando se solicitó la información.

Comportamiento del recaudado por concepto de impuesto departamental de degüello de ganado mayor en el Municipio de Montería en los años 2013-2018

\section{RECAUDO IMPUESTO DEGUELLO DE GANADO MAYOR VIGENCIAS 2014 - 2019}

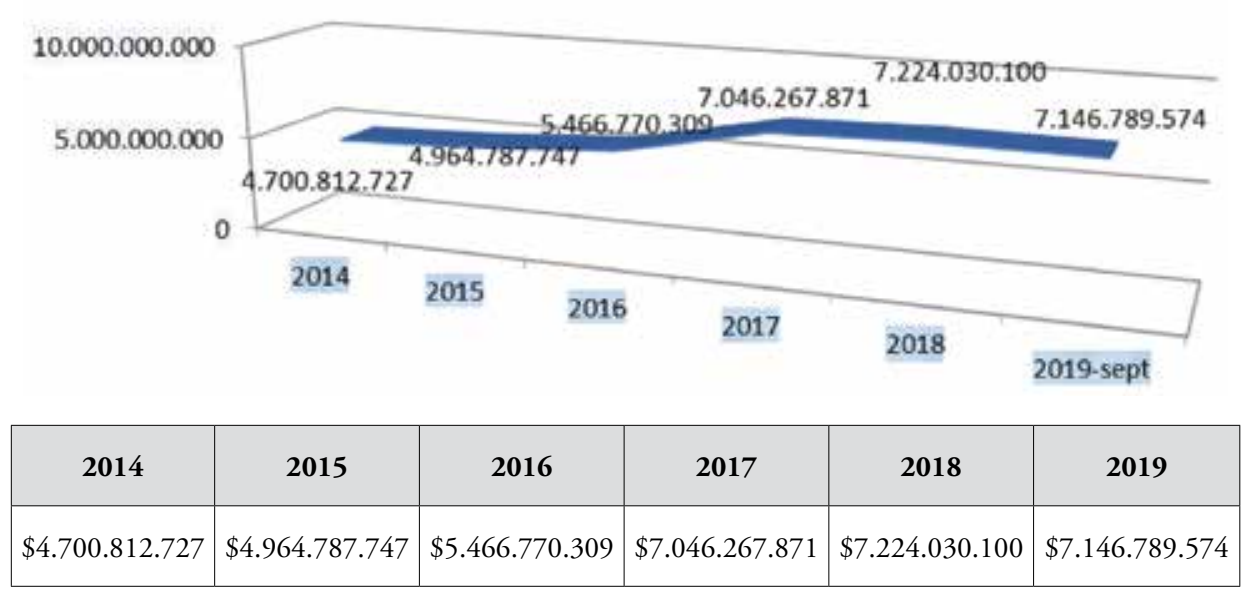

Se puede decir que otras causas que afectan el recaudo de este impuesto es la clandestinidad en el sacrificio del ganado, no todos los bovinos son movilizados al frigorífico autorizado.

De la misma problemática periódicos locales del Departamento se han pronunciado. Uno de estos, el periódico virtual La Razón (2018), señaló que al menos el 60\% de la carne consumida en Córdoba proviene de mataderos clandestinos. En 2018 la Facultad de Zootecnia y Medicina Veterinaria de la Universidad de Córdoba reveló que más de la mitad de la carne comercializada en esta sección del país proviene de mataderos clandestinos, sin la mínima condición de higiene; al respecto, Nicolás Martínez Humanes decano de la Facultad de Medicina Veterinaria de la Universidad de Córdoba señaló que la carne sin control sanitario genera un grave riesgo para la salud de las personas que la consumen, sobre todo porque en Córdoba hay antecedentes de enfermedades transmisibles, como rabia y brucelosis.

El recaudo del impuesto al degüello de ganado mayor en el Municipio de Montería, no se analiza de manera individual, el cual es el objeto de estudio de este proyecto; 
según la oficina de Secretaría de Hacienda Departamental, no pudo individualizar la cifra recaudada por ese municipio, aduciendo que por ser Frigosinú el único matadero en Montería, es información de carácter reservada, según la (Ordenanza 07, 2012, art. 379) que acredita la información tributaria departamental con carácter reservado y de conformidad con el Decreto 624 de 1989, artículo 583 del Estatuto Tributario "la información tributaria respecto de las bases gravables y la determinación privada de los impuestos que figuren en las declaraciones tributarias, tendrán el carácter de información reservada”; por lo anterior la Gobernación solo puede dar cifras impersonales de las estadísticas. En el Departamento de Córdoba existen cinco mataderos de bovinos legalmente constituidos y que se encuentran abiertos, los cuales son: en Montería Frigosinú, en Cereté Frigocer-Expocol, en Valencia Matadero Municipal de Valencia, en Montelíbano Planta de Beneficio Municipal Jaguazul, en Ciénaga de Oro Red Cárnica; de los cuales no se pudo obtener información individualizada de los recaudos por la razón antes mencionada. Dicho lo anterior con respecto al Municipio, es importante resaltar y analizar la participación que tuvo el impuesto al degüello de ganado mayor en el Departamento de Córdoba frente a lo recaudado a los demás ingresos tributarios departamentales, en los años de estudio 2013 a 2018.

Al comparar el recaudo del impuesto al degüello de ganado mayor con los demás tributos y ordenarlos en orden descendente de mayor a menor recaudo por ańo, se puede observar que para la vigencia 2014 el degüello de ganado mayor ocupo el noveno lugar y para la vigencia 2018 se posicionó en el octavo lugar; es decir, de los trece tributos departamentales se posiciona en lugares de inferior recaudo en relación con los primeros puestos que ocupa el consumo de cervezas y licores, siendo Córdoba un departamento reconocido por la ganadería en los ámbitos nacional e internacional. Cabe resaltar que la Secretaría de Hacienda, que es el ente recaudador, debe hacer mayor control al impuesto al degüello de ganado para evitar la evasión en lugares inhóspitos. Otra de las razones por las cuales es bajo el recaudo en Montería es por el alto costo del impuesto con respecto al de otros municipios.

Se dirigió petición a la empresa Frigosinú en la oficina de gerencia de talento humano en varias ocasiones y como respuesta el gerente no autorizó la visita para dar declaraciones de las funciones de Frigosinú. En las otras entidades con las que sí se logró hacer las entrevistas resultó que existen muchos mataderos clandestinos, lo cual es preocupante porque no existen medidas sanitarias y ambientales para el sacrificio del ganado mayor, que perjudica a quienes finalmente consumen el producto. El funcionario de la cvs en la entrevista esbozó que en Palo de Aguas, Lorica y Sahagún había mataderos que eran ilegales desde el punto de vista ambiental, los cuales se cerraron, en este último se generaba la quema de huesos.

La Policía Ambiental y Ecológica maneja la información sobre inspección de la zona urbana y rural para identificar los mataderos clandestinos, estos tienen facultades para realizar operativos, investigar y decomisar carne no apta para el consumo y seguir el conducto regular para los establecimientos ilegales; pero no se logró obtener respuesta pues se negaron a dar declaraciones. El proceso de fiscalización y recaudo 
del impuesto al degüello de ganado mayor en Córdoba lo adelanta la Secretaría de Hacienda Departamental, que gestiona los ingresos tributarios de orden departamental.

Comparativos recaudos de impuesto del periodo 2014-2018

\begin{tabular}{|c|c|c|c|}
\hline Impuestos & Vigencia 2014 & Impuesto & Vigencia 2018 \\
\hline $\begin{array}{l}\text { Consumo de cervezas, } \\
\text { mezclas y refajos }\end{array}$ & $\$ 59.279 .043 .000$ & $\begin{array}{l}\text { Consumo de cervezas, } \\
\text { mezclas y refajos }\end{array}$ & $\$ 80.161 .504 .920$ \\
\hline $\begin{array}{l}\text { Consumo de licores, } \\
\text { vinos y aperitivos }\end{array}$ & $\$ 28.592 .790 .000$ & $\begin{array}{l}\text { Consumo de licores, } \\
\text { vinos y aperitivos }\end{array}$ & $\$ 34.851 .537 .000$ \\
\hline Estampillas & $\$ 17.231 .591 .295$ & Estampillas & $\$ 18.269 .727 .788$ \\
\hline Impuesto de Registro & $\$ 12.486 .913 .961$ & $\begin{array}{l}\text { Consumo de cigarrilos } \\
\text { y tabaco elaborado }\end{array}$ & $\$ 16.485 .998 .001$ \\
\hline Sobretasa a la gasolina & $\$ 11.028 .299 .000$ & Impuesto de Registro & $\$ 16.480 .335 .139$ \\
\hline Vehículos automotores & $\$ 8.951 .397 .423$ & Sobretasa a la gasolina & $\$ 14.276 .709 .970$ \\
\hline $\begin{array}{l}\text { Consumo de cigarrilos } \\
\text { y tabaco elaborado }\end{array}$ & $\$ 8.313 .930 .000$ & Vehículos automotores & $\$ 13.890 .056 .565$ \\
\hline $\begin{array}{l}\text { Estampillas para el bienestar } \\
\text { adulto mayor }\end{array}$ & $\$ 6.650 .685 .910$ & Degüello de ganado mayor & $\$ 7.224 .034 .100$ \\
\hline Degüello de ganado mayor & $\$ 4.700 .812 .727$ & $\begin{array}{l}\text { Estampillas para el bienestar } \\
\text { adulto mayor }\end{array}$ & $\$ 6.650 .685 .910$ \\
\hline $\begin{array}{l}\text { Estampilla prodesarrollo } \\
\text { departamental }\end{array}$ & $\$ 4.531 .263 .622$ & $\begin{array}{l}\text { Estampillas prouniversidad } \\
\text { pública }\end{array}$ & $\$ 4.890 .460 .025$ \\
\hline $\begin{array}{l}\text { Estampillas prouniversidad } \\
\text { pública }\end{array}$ & $\$ 3.966 .926 .531$ & $\begin{array}{l}\text { Estampilla prodesarrollo } \\
\text { departamental }\end{array}$ & $\$ 4.629 .215 .645$ \\
\hline Estampilla procultura & $\$ 1.383 .759 .369$ & Estampilla procultura & $\$ 1.504 .181 .894$ \\
\hline $\begin{array}{l}\text { Estampilla proelectrificacion } \\
\text { rural }\end{array}$ & $\$ 698.955 .863$ & $\begin{array}{l}\text { Estampilla proelectrificacion } \\
\text { rural }\end{array}$ & $\$ 183.771 .830$ \\
\hline Total & $\$ 150.584 .777 .406$ & Total & $\$ 201.639 .903 .483$ \\
\hline
\end{tabular}

En el comienzo de este trabajo se planeó visitar algunas entidades ambientales, sanitarias y la Planta de Sacrificio del Municipio de Montería, así como cvs, ICA e Invima, en las cuales, por sus competencias y funciones con el tema de investigación, se obtuvo información muy valiosa para este artículo conforme a los objetivos propuestos; no obstante, en otras como Frigosinú, Policía Ambiental, Secretaría de Salud, pese a varias peticiones realizadas fueron negadas las solicitudes de las entrevistas para rendir declaraciones. Se analizaron las declaraciones obtenidas en cada entidad para determinar su participación en el control de mataderos clandestinos. 


\section{Corporación Autónoma Regional de los Valles del Sinú y del San Jorge (CVS)}

Se realizó entrevista a un funcionario encargado del área control de desechos y residuos peligrosos, Frigosinú representa el establecimiento con mayor generación de residuos peligrosos al ambiente según informe final plan de manejo integral de residuos o desechos peligrosos del Departamento de Córdoba.

La cvs es una autoridad ambiental que autoriza o no la realización de actividades que impactan ambientalmente a la región, su cobertura geográfica en el Departamento de Córdoba es: Alto Sinú: Tierra Alta, Valencia, Sinú Medio: Montería, Cereté, Ciénaga de Oro, San Carlos, San Pelayo, Bajo Sinú: Lorica, Purísima, Momil, Chima, Cotorra, Sabanas; Sahagún, Chinú, Pueblo Nuevo, San Andrés de Sotavento, San Jorge; Planeta Rica, Montelíbano, La Apartada, Buenavista, Puerto Libertador, Ayapel, Costanera; San Antero, San Bernardo del Viento, Moñitos, los Córdobas, Canalete, Puerto Escondido.

Referente al sacrificio de ganado, la cvs controla las actividades de las plantas de beneficio que puedan generar impacto al medio ambiente; en caso de que haya violación de las normas de protección ambiental, existen procesos sancionatorios como cierre del establecimiento, así como lo comentó el funcionario en la entrevista, en los casos que se presentaron en Palo de Aguas, Lorica y Sahagún, que dichos mataderos eran ilegales desde el punto de vista ambiental, estos se cerraron.

Desde la Corporación se hace seguimiento y control de las actividades que realiza Frigosinú, este es el único matadero legalmente constituido de Montería y los desechos generados por el matadero son vertidos en la laguna de oxidación. En el Departamento hubo varias quejas años atrás por el mal olor de la laguna generado por los desechos que se vierten del sacrificio de ganados, equinos y asnos; las quejas que se presentan de los establecimientos son pocas, de tres a cuatro al año. Los permisos que exige la cvs para que los mataderos puedan operar en la parte ambiental son permisos de vertimiento que cuenten con sistema de agua residual, manejo de residuos sólidos; si tratan sangre y orina se deben tener permisos atmosféricos; para los establecimientos de mataderos requieren de un plan de manejo ambiental.

La actividad de sacrificio genera residuos (sangre y orina) que se vierten en la laguna de oxidación (biodegradación de materia orgánica), son sustancias proteicas, que generan olores característicos. La laguna queda cerca de Frigosinú en un sector llamado Furatena, cuando hay lluvias y pasa por los complejos lagunares esta se desborda, generando quejas frecuentes; las mismas lluvias hacen que el agua en la laguna se revuelva y se agite y por ende salen los gases con olores; en Frigosinú después de tratar esa materia orgánica en la laguna de oxidación, se manda al alcantarillado el agua residual tratada de su actividad, ya cumpliendo una normatividad de límite de vertimiento.

En esta investigación surge un interrogante: ¿los desechos generados por la laguna de oxidación y los vertederos afectan a largo plazo el ecosistema o son desechos biodegradables? El llamado sistema de tratamiento de aguas residuales es 
el proceso en el que se realiza un seguimiento, se extraen los lodos y se les hacen unos análisis de peligrosidad y según los resultados se les hace tratamiento. Cuando hacen la extracción del lodo de la laguna es porque ha llegado el tiempo en que la laguna pierde su vida útil, se extrae y tienen un hecho de secado, la tratan y lo mezclan junto con el rumen (cámara de fermentación o saco que tienen los bovinos en el estómago); y es algo que se llama 'compost' o derramamiento de suelo, para favorecer la recuperación de suelos, y resulta muy útil como fertilizante natural; el periodo de vida útil de la laguna es de veinte años, pero cuando aumenta el número de reses sacrificadas le realizan mantenimiento más rápido.

El residuo de la sangre producto de la muerte del ganado lo almacenan y hacen harinas para venderlas a fabricantes de concentrados de animales; cuando toda la sangre no se recoge queda en el piso, con el tiempo se va biodegradando y son estos los líquidos que van a la laguna.

\section{Instituto Colombiano Agropecuario (ICA)}

Se realizó entrevista a dos funcionarios en el área de Sanidad animal, subáreas de Epidemiologia e Inocuidad Pecuaria. El ICA se encarga de vigilar la productividad de actividad primaria de bovinos, porcinos, obimoprimos, peces, parte de avicultura, es decir, la crianza de estos animales en el campo, alimentación, vacunas y toda la parte sanitaria.

En lo que concierne a la actividad ganadera, el entorno en el que se cría el animal debe estar en buenas condiciones, con espacio para movilizarse, alimento, pasto y agua limpia; además, en la parte de enfermedades y vacunación, proteger al ganado con un control oficial, que son: aftosa, brucelosis, tuberculosis, rabia y estomatitis vesiculares.

En las declaraciones los funcionarios que se entrevistaron hablaron de la intervención del ICA en las plantas de sacrificios de ganado cuando hay una notificación por sospecha de una enfermedad de control oficial. Cuando ingresan animales muertos, que se mueren en el camión o llegan a la planta de sacrificio y mueren allí. Pero quienes tienen el control de esos animales desde que ingresan a la planta hasta que realizan todo el proceso del sacrificio son los funcionarios de la planta y del Invima.

El ICA realiza visitas y seguimientos de ingreso de animales para sacrificar en fincas de la región, los funcionarios van por días a las plantas de sacrificio y controlan el ingreso. Cuando se realizan visitas a los predios se mira que los animales cumplan con la vacunación, que todas las movilizaciones se hagan con licencia de ingreso, salida y control de nacimientos, para que el predio obtenga certificación (permiso sanitario) como productor de carne.

El ica solo llega hasta el ingreso de los animales, ahí se mide la eficiencia y la eficacia del transportador, desde que lleguen con su licencia hasta que cumplan con todas las características, integridad del animal y vacunación.

En las fincas cuando es ciclo completo: cría, levante, ceba, en estas fincas cebadoras salen los animales con destino a sacrificio, para consumo humano. Salen de 
la finca y entran al transporte, entonces es responsabilidad de esta persona, que le ofrezcan también confort, bienestar al animal, porque es en el transporte donde más se dañan, pues en el viaje a la planta de beneficio llega el animal con trauma, golpes y cuando el animal es sacrificado que se le quita el cuero se encuentran con los moretones y la carne negra, aquí se efectúa el decomiso por la carne afectada.

La Policía debe velar por que los transportistas tengan la guía de movilización de los animales (carácter de que lo que se transporta es legal).

A la llegada del ganado al frigorífico intervienen los entes territoriales, salud pública y el Invima, esta última entidad se encarga del empaque en condiciones higiénicas y de que la carne sea apta para el consumo humano.

La Policía de Córdoba detectó una planta de sacrificio que operaba clandestinamente en el municipio de Montelíbano. Según reportes de las autoridades, las reses eran sacrificadas sin contar con las normas mínimas de higiene, se incautaron en el lugar 3120 kilos de carne de res evaluados en $\$ 20.970 .000$, los cuales, al encontrarse sin condiciones de salubridad para el consumo humano, fueron puestos a disposición de la Secretaría de Salud para su destrucción; en otros municipios también hay establecimientos clandestinos operando de la misma manera, situación muy preocupante pues los habitantes serán los más afectados al consumir carne que ya perdió su inocuidad (Contexto Ganadero, 2013).

\section{Instituto Nacional de Vigilancia de Medicamentos y Alimentos (Invima)}

En esta entidad se realizó la entrevista a la gerente encargada de controlar y supervisar algunas áreas de salud pública. Con respecto a la pregunta relacionada al seguimiento y control que ejerce sobre los mataderos en Montería la respuesta que se obtuvo fue que su competencia es exclusiva inspección de vigilancia y control de la producción, procesamiento de alimento de las plantas de beneficio de animales de los centros de acopio, así como el transporte asociado; en el Invima hay unos inspectores oficiales dentro de las plantas.

Para mayor claridad, la funcionaria citó el siguiente ejemplo: un empresario privado que quiere iniciar una planta de beneficio animal necesita autorización y la expide el Invima, el decreto más importante es el 1500 recientemente modificado por el Decreto 1775/2019; el empresario prepara sus documentos, sus instalaciones y se comunica con el Invima para que realice la visita para ser certificado. Una vez está autorizada la planta, debe solicitar al Invima la inspección oficial; en una planta como Frigosinú es permanente el inspector oficial.

También se dio respuesta a otros interrogantes como ¿cuáles son los mataderos en Montería legalmente constituidos?, se hizo referencia a que el Invima trabaja bajo enfoque de predios:

- Plantas de bovinos en Montería Frigosinú (ganado mayor)

- Planta Porciavicola Pineda en Montería (ganado menor) 
Por otra parte, la funcionaria señaló los mataderos existentes en el Departamento de Córdoba, en Montería (Frigosinú), en Ciénaga de Oro (Athenafoods compañía de red cárnica), en Cereté (Frigocer-Expocol); planta de beneficio en Montelíbano (Jaguazul), planta municipal en Valencia; la planta municipal de Lorica se encuentra cerrada (FrigoLorica-Coproexcal). La única planta para sacrificio de cerdo en la región se encuentra en Sampués (Frigorífico La Bananera).

El inspector oficial del Invima se encuentra de forma permanente, además de Frigosinú Montería, en Frigocer-Expocol Cereté y Red Cárnica Ciénaga de Oro. Y de manera periódica, con visitas que programa, en la Planta de Beneficio Municipal de Montelíbano y Valencia.

Las plantas de sacrificio cerradas son FrigoLorica-Coprocoexcal, Comercializadora de Ganado Casagrande en Sahagún y Planta Municipal de Ayapel. En el departamento están en proceso de construcción las siguientes plantas de beneficio para ganado mayor: en Ciénaga de Oro (Cárnicos del Caribe), San Carlos (Encarnes) y Tierralta (Frigorífico del Alto Sinú).

El Invima interviene en todos los procesos, verifica que estén realizando la normatividad desde que llegan los bovinos a las plantas intervinientes, pero no tiene contacto con los ganaderos; y garantiza la inocuidad (apto para el consumo humano). Intervienen por equipos de inspección, arranca la inspección en el proceso antemortem, es decir, antes de que se muera el animal, llegan a las plantas de beneficio, hay unos corrales donde los animales deben permanecer en reposo un mínimo de seis horas para que en ese reposo se manifiesten síntomas de posibles enfermedades, que permitan a los inspectores oficiales (que son médicos veterinarios) poder observar dichos síntomas; se hace el llamado al ICA y se aíslan en un corral diferente. Con los animales aptos para el sacrificio el inspector vigila el proceso de insensibilización (hay una pistola le disparan al hueso central del bovino y este queda insensibilizado), proceden con el sacrificio hasta finalmente ser producto cárnico, el Invima también vigila cuando lo suben en un vehículo destinado a expendios y carnicerías. La planta de Frigosinú es una planta de exportación, el producto va empacado al vacío y que el producto cumpla con la actividad sanitaria de otros países.

También está en la inspección de vísceras; por ejemplo, si encuentran pulmones o hígados u otro tipo de órgano del ganado que presentan sintomatología de alguna enfermedad se decomisan, se destruyen y se deja constancia del decomiso.

Se puede decir que los funcionarios entrevistados en las diferentes entidades se abstienen de dar información o señalar datos precisos que dejen en evidencia mataderos clandestinos, aunque sepan de su práctica ilegal. Relacionaremos las actividades, los procesos o funciones en los que intervienen las entidades que no rindieron declaraciones en cuanto al sacrificio de degüello de ganado mayor. Frigosinú es el único matadero legalmente constituido de la ciudad de Montería; la Policía Ambiental es la autoridad ambiental que controla el funcionamiento de las plantas ilegales e incauta el producto cárnico del ilícito. Lo referente al transporte, distribución y comercialización desde las plantas de procesamiento hasta los puntos donde se comercializan 
y el expendio de los productos en restaurantes, tiendas, supermercados está bajo la Autoridad Sanitaria del Departamento.

A pesar de que las entidades se abstengan de hablar de establecimientos ilegales, traigo a colación la siguiente noticia: "En bodega clandestina de Montería se pudrieron 8 toneladas de carne, la Policía Ambiental desmanteló establecimiento que guardaba carne en condiciones inapropiadas, sin contar con la debida refrigeración y almacenamiento, los residentes del sector denunciaron la situación; la Secretaría de Salud, Martha Ruiz, aseguró que el establecimiento es ilegal, pues llegaron al lugar solicitaron los permisos pertinentes éstos no fueron entregados. Aducían que 'La carne llegó de una planta de sacrificio en Ciénaga de Oro era de muy buena calidad, el problema estuvo en la baja capacidad que tenían para refrigerarla'; ante esto, la autoridad procedió a incinerar la carne” (Contexto Ganadero, 2013).

\section{Conclusiones}

1. Con las entrevistas realizadas se logró identificar que en el Municipio de Montería existe solo una planta de sacrificio autorizada, de la cual no se obtuvo análisis de recaudo por ser información reservada, sino que la Secretaría de Hacienda Departamental de Córdoba proporcionó el recaudo de las cinco plantas legales que contribuyen; por otra parte, existen otros lugares donde se ejercen las mismas actividades con la diferencia de que no cumplen con condiciones de salubridad y no contribuyen con el pago del impuesto, es decir, existe ineficiencia en su recaudo por factores como la falta de operativos por parte de las autoridades como la Policía Ambiental, la Secretaría de Salud, la Secretaría de Hacienda. Muchos casos también se dan por desconocimiento, pues los ganaderos sacrifican el animal en la misma finca en que lo crían y luego venden la carne; no se dan por enterados del impuesto. En Montería, por ser la capital ganadera del país, el recaudo, según las estadísticas en el año 2018, ocupa el octavo lugar de los trece tributos departamentales, con una cifra de \$7.224.034.100; se posiciona en lugar muy inferior de recaudo en relación con el primer puesto, ocupado por el consumo de cervezas y licores.

2. Se puede decir que existe alta evasión del impuesto al degüello de ganado en la región. En repetidas ocasiones se intentó tener una entrevista con entidades como Frigosinú, la Secretaría de Salud, la Policía Ambiental, las cuales son entes directamente vinculados en la materia de estudio de este artículo, y se negaron a dar declaraciones; por el contrario, se lograron entrevistas con otras entidades, las cuales fueron ICA, CVs e Invima. Las primeras no quisieron dar declaraciones y las segundas se abstienen de acusar o hacer referencia a los mataderos clandestinos, dicen que no manejan información referente a la práctica ilegal de mataderos o que son pocos los casos registrados, a pesar de que los noticieros locales informan que sí existe el ilícito. Intentan camuflar una problemática que la comunidad no desconoce, pero es a las entidades con sus facultades y autoridad a las que corresponde controlar los sitios clandestinos de sacrificio de ganado. 
3. Si por evasión la Secretaría de Hacienda de Córdoba deja de recaudar tributos se perjudica la población; por el contrario, se requiere fortalecer las rentas departamentales para la reinversión social en el municipio y todo el departamento, por ende esta investigación ofrece apoyo a la Dirección de Rentas Departamentales de Córdoba y municipal de Montería, en cuanto a mejorar las gestiones que se puedan adelantar para mejorar el recaudo del impuesto de ganado mayor, el cual debería ser el fuerte debido a la actividad de ganadería en la región; se propone la creación de un grupo anticontrabando o anticorrupción e incluirlo en la ordenanza que expide el Departamento, en el cual trabajen de la mano todas las autoridades que están relacionadas, en pro del recaudo de impuestos locales (policía metropolitana y demás secretarías del Departamento y Municipio), para que las rentas del Departamento se fortalezcan debido a que son muy afectadas por la evasión y se dejan de percibir ingresos importantes, que podrían destinarse para reinvertirlos en áreas como educación y bienestar social; dicho grupo ya se ha creado en otros departamentos del país (como Antioquia, Cundinamarca, Valle del Cauca) y ha empezado a ser exitoso.

4. El Departamento debe implementar medidas para que se pueda controlar toda la evasión que existe; con los recursos para la operatividad y procesos de recaudo no se logra controlar y vigilar todos los establecimientos clandestinos e ilegales de sacrifico de ganado en la región, ocasionando grandes pérdidas de recursos que podrían convertirse en salud, educación, vivienda para todos los cordobeses.

\section{REFERENCIAS BIBLIOGRÁFICAS}

Aldana, U., Alonso, T. H., \& Albarracín, M. M. (2019). Influencia de los índices asociados a la corrupción en el desarrollo económico departamental en Colombia. Apuntes Contables.

Ardila, S. (20 de junio de 2019). Análisis del impuesto al degüello de ganado en el departamento del Tolima de los años 2014-2018. Obtenido de https://repository.ucc.edu.co/ handle/20.500.12494/11687.

Artículo 583 (31 de diciembre de 2019). Estatuto Tributario Nacional. Bogota, Colombia.

Betancur, L. F. (octubre de 2007). ESAP: Las finanzas públicas territoriales utopia o realidad caso proceso de tributación en el Municipio de Taminango - Nariño. Obtenido de http://cdim.esap.edu.co/bancomedios/documentos\%20pdf/las\%20finanzas $\% 20$ p\%C3\%BAblicas\%20territoriales\%20utopia\%20o\%20realidad.pdf.

Carrascal, A. (19 de noviembre de 2015). Sistema de recaudo de los Impuestos del Municipio de Ocaña. Obtenido de repositorio.ufpso.edu.co:8080/dspaceufpso/handle/123456789/766.

Contexto Ganadero. (4 de noviembre de 2013). Contexto ganadero. Obtenido de https:// www.contextoganadero.com/regiones/sigue-sin-freno-el-sacrificio-ilegal-en-el-pais. Congreso de Colombia (30 de marzo del 1989) Articulo 583 [título ir]. Estatuto Tributario de los Impuestos Administrados por la Dirección General de Impuestos Nacionales. [Decreto 624 de 1989]. DO: 38.756. 
Contexto ganadero (18 de enero de 2013). Contexto ganadero. Obtenido de https://www. contextoganadero.com/regiones/en-bodega-clandestina-de-monteria-se-pudrieron8 -toneladas-de-carne.

Contexto ganadero (6 de febrero de 2013). Contexto ganadero. Obtenido de https://www. contextoganadero.com/regiones/policia-desmantela-planta-de-sacrificio-ilegal-enmontelibano.

Coronel, L. M., \& Pérez, Y. (19 de noviembre de 2015). Cultura de la poblacion colombiana con la contribucion del impuesto predial. Obtenido de repositorio.ufpso.edu.co:8080/ dspaceufpso/handle/123456789/769.

Decreto 1222 (18 de abril de 1986). Código de Régimen Departamental. Bogotá, Colombia. El Tiempo (7 de septiembre de 1993). Periodico el tiempo. Obtenido de https://www. eltiempo.com/archivo/documento/MAM-216067.

Farfán, J. C., \& Mosquera, Y. L. (2015). Propuesta metodológica para aumentar el recaudo tributario en el municipio de Quibdó. Contaduría Universidad de Antioquia (66), 81-112.

Fedegan, F. C. (10 de marzo de 2019). Bases para la formulación del plan de acción 20142018 para el mejoramiento de la ganadería del departamento de Córdoba. Federación Colombiana de Ganaderos (Fedegán). Obtenido de file://E:/sEminario\%20DE\%20 GRADO/IMPTO\%20DEGUELLO/trabajos\%20para\%20revision\%20de\%20literatura/2. PlanCordobafinal\%20(fedegan).pdf.

González, J. C., \& Salazar, G. (15 de abril de 2008). Proyecto fortalecimiento de la actividad artesanal. Familias guardabosques. Tierralta, Córdoba. Obtenido de Ministerio de Comercio Industria y Turismo: https://repositorio.artesaniasdecolombia.com.co/ handle/001/2033.

Henao, V. M., \& Caro, A. D. (2019). Valoración por el método de flujo de caja libre: finca bananera de Urabá. Apuntes Contables.

Hoyos, Y. V. (20 de octubre de 2016). Biblioteca Digital Universidad del Valle: aspectos de las carnicerias en Cali en los años 70 y 80 del siglo XIX. Obtenido de http://hdl.handle. net/10893/9870.

La Razón (2018). Periódico virtual la razón. Obtenido de https://larazon.co/temas-del-dia/almenos-el-60-de-la-carne-consumida-en-cordoba-proviene-de-mataderos-clandestinos/.

Lindo, M. E. (Julio de 2016). Universidad Militar Nueva Granada: determinar las estrategias para el incremento del recaudo tributario. Obtenido de https://repository.unimilitar. edu.co/bitstream/handle/10654/14558/LindoSanchezMauricioEnrique2016.pdf;j sessionid=C96181FC2B7FCD6A65E5C5 СввC8424E1 ?sequence $=1$.

Loaiza, M. B., Cardona, A., \& Quintero, D. C. (11 de mayo de 2012). Comparativo del código de rentas de los municipios de Pereira y Dosquebradas en los impuestos territoriales. Obtenido de http://repositorio.unilibrepereira.edu.co:8080/pereira/bitstream/ handle/123456789/691/COMPARATIVO\%20DEL\%20CODIGO\%20DE\%20rentas. pdf?sequence $=1$.

López, J. A. (10 de julio de 2014). Análisis estratégico de los tributos departamentales mediante modelamiento y simulación utilizando Dinámica de Sistemas. Obtenido de http://b digital.unal.edu.co/39495/. 
Mosquera, A. (2014). Biblioteca Digital Universidad del Valle: Análisis de la administración de la politica fiscal y sus efectos en el nivel de competitividad, en el Municipio de Rio Sucio-Caldas en el periodo 2008-2013. Obtenido de http://bibliotecadigital.univalle. edu.co/bitstream/10893/9133/1/CB-0519731.pdf.

Núñez, J. (1. ${ }^{\circ}$ de agosto de 2005). Diagnóstico básico de la situación de los ingresos por impuestos del orden municipal en Colombia. Obtenido de Universidad de los Andes: https://ideas.repec.org/p/col/000089/002343.html.

Perea, R. N., Palacios, K., \& Rentería, F. (2018). Desarrollo de una estrategia para el mejoramiento del recaudo del impuesto de degüello de ganado mayor en el departamento de Chocó.

Pérez, Z. A. (31 de octubre de 2016). Universidad de Cordoba: Validación de limpieza y desinfección de manos, guantes acerados de la sala de deshuese y el punto crítico de control desinfección de canales de la empresa Frigosinú S. A. Obtenido de https://repositorio. unicordoba.edu.co/handle/123456789/1042.

Restrepo, J. J., Hernández, B., \& Moreno, N. J. (2013). Evaluacion de la eficiencia en el recaudo del impuesto al defuello de ganado mayor, sacrificado en el Municipio de Florencia, en los periodos fiscales 2009-2010. FACCEA.

Talero, K. A., \& Quintero, L. V. (2011). Comparativo entre los impuestos más representativos de los departamentos de Quindio y Valle del Cauca. Obtenido de Universidad Libre: http:// repositorio.unilibrepereira.edu.co:8080/pereira/bitstream/handle/123456789/571/ COMPARATIVO\%20ENTRE\%20LOS\%20IMPUESTOS\%20M\%C3\%81S\%20REPRESENTATIVOS\%20DE\%20LOS\%20DEPARTAMENTOS\%20DE\%20\%20QUINDIO\%20Y\%20 VALLE $\% 20$ DEL $\% 20$ CAUCA.pdf? sequence $=1$.

Valencia, W. R. (12 de agosto de 2017). Análisis de la información tributaria del departamento de Arauca para el mejoramiento en el recaudo por parte de la Secretaría de HaciendaGobernación de Arauca. Obtenido de http://hdl.handle.net/20.500.12494/10167.

Vasquez, M. D., \& Jiménez, L. K. (21 de mayo de 2015). Análisis del comportamiento tributario de los comerciantes en el sector del mercado público de la ciudad de Ocaña, Norte de Santander. Obtenido de repositorio.ufpso.edu.co:8080/dspaceufpso/ handle/123456789/624. 